\title{
Nonlinear dynamics of short traveling capillary-gravity waves
}

\author{
C. H. Borzi, ${ }^{1}$ R. A. Kraenkel, ${ }^{2}$ M. A. Manna,${ }^{3}$ and A. Pereira ${ }^{3}$ \\ ${ }^{1}$ Facultad de Ciencias Exactas y Naturales de la Universidad Nacional de Buenos Aires, Pab. II, Nuñez, Buenos Aires, Argentina \\ ${ }^{2}$ Instituto de Física Teórica-UNESP Rua Pamplona 145, 01405-900 São Paulo, Brazil \\ ${ }^{3}$ Physique Mathématique et Théorique, CNRS-UMR5825, Université Montpellier II, 34095 Montpellier, France \\ (Received 16 July 2004; revised manuscript received 29 November 2004; published 23 February 2005)
}

\begin{abstract}
We establish a Green-Nagdhi model equation for capillary-gravity waves in $(2+1)$ dimensions. Through the derivation of an asymptotic equation governing short-wave dynamics, we show that this system possesses $(1+1)$ traveling-wave solutions for almost all the values of the Bond number $\theta$ (the special case $\theta=1 / 3$ is not studied). These waves become singular when their amplitude is larger than a threshold value, related to the velocity of the wave. The limit angle at the crest is then calculated. The stability of a wave train is also studied via a Benjamin-Feir modulational analysis.
\end{abstract}

DOI: 10.1103/PhysRevE.71.026307

PACS number(s): 47.10.+g, 47.20.Ky, 47.35.+i, 04.25.-g

\section{INTRODUCTION}

The propagation of surface waves in an ideal incompressible fluid is a classical and still open subject of investigation in fluid mechanics. One possible approach to tackle this problem is to elaborate approximate theories from hypotheses on the nature of the waves. Thus, the shallow water approximation has produced a lot of interesting and useful nonlinear evolution model equations for small-amplitude long surface waves. These models can be classified in two main categories: extreme long-wave models like the Korteweg-de Vries (KdV) or the modified KdV (mKdV) equations [1,2], and intermediate long-wave models like the various versions of the Boussinesq or modified Boussinesq equations [3], the Benjamin-Bona-Mahony-Peregrine equation [4,5], the Green-Nagdhi system [6-8], and many others. Roughly speaking, extreme models may be derived from intermediate ones since the latter allow an asymptotic limit leading to the ubiquitous $\mathrm{KdV}$ or $\mathrm{mKdV}[9,10]$. With regard to long-wave dynamics, intermediate models are therefore more precise concerning dispersion and nonlinearities, and accordingly are more representative of Euler systems than extreme ones. However, this richer description of the waves has a counterpart for it may incorporate also short-wave propagation in the models. It is due to the methods leading from the Euler system to the intermediate models which are not able to filter out completely short waves. This is the case, for example, of some of the Boussinesq-type equations and the Benjamin-Bona-Mahony-Peregrine equation $[10,11]$.

The presence of short scales in a long-scale model may constitute a major drawback, especially during its numerical study. Since in that case the final dynamics are a nonlinear superposition of short and long scales, the short waves if unstable can contaminate the whole model. For this reason, nonlinear propagation of short waves in long-wave models has been previously studied in Refs. [12-15]. Linear theoretical estimates on the behaviors of short waves were carried out, based on the linear dispersion relations of the models and thus confirmed by numerical tests performed on the nonlinear models. A nonlinear analytical and numerical analysis of this question for the Benjamin-Bona-Mahony-Peregrine equation was conducted in Ref. [11]. However, we should not reduce such dynamics to a subsidiary phenomenon, since their study is a way to understand the ultraviolet regime in surface water wave.

The purpose of this paper is to investigate theoretically and numerically nonlinear short-wave behavior in a GreenNagdhi model with surface tension. Although the model is derived in a limit where large scales dominate, the existence of short waves related to the short scale nature of the capillary phenomena cannot be ignored. The study is carried out in $(2+1)$ dimensions. In the long-wave limit, the model leads to the Kadomtsev-Petviaskvili (KP) equation. Here, we have only considered short waves since, at present, we lack the tools needed to take into account both scales together. This is an important open problem and some progress was made in Refs. [16,17].

The paper is organized as follow. In Sec. II, we derive a Green-Nagdhi system in $(2+1)$ dimensions, with surface tension. The analysis of the associated linear dispersion relation shows that the model can propagate short waves. To tackle the problem of nonlinear short surface waves, a multiple scale perturbative method is carried out in Sec. III and leads to an asymptotic model equation. The analytical study of its $(1+1)$ traveling-wave solutions is then undertaken in Sec. IV and, finally, we perform in Sec. V the Benjamin-Feir analysis of the Stokes wave. The last section is devoted to some final remarks.

\section{THE (2+1) GREEN-NAGDHI MODEL EQUATION WITH SURFACE TENSION}

Let us consider a fluid layer, initially at rest, in a uniform gravitational field and endow the space with a Cartesian frame $(O, x, y, z)$ so that $(O z)$ is the upward vertical direction. The fluid domain is contained between a rigid bottom at $z=0$ and a upper free surface at $z=S(x, y, t)$. We assume that the fluid is ideal, i.e., inviscid, incompressible, and that its density, $\sigma$, is uniform and constant. Its surface tension is denoted by $T$ and the velocity field by $\mathbf{v}=(u, v, w)$, where each component depends on $x, y, z$, and $t$. The motion of the fluid in the bulk is then given by the Euler equations. For $0<z<S(x, y, t)$, we have 


$$
\begin{gathered}
u_{x}+v_{y}+w_{z}=0, \\
u_{t}+u u_{x}+v u_{y}+w u_{z}=-p_{x}^{*} / \sigma, \\
v_{t}+u v_{x}+v v_{y}+w v_{z}=-p_{y}^{*} / \sigma, \\
w_{t}+u w_{x}+v w_{y}+w w_{z}=-p_{z}^{*} / \sigma-g,
\end{gathered}
$$

where $p^{*}$ is the pressure field in the fluid and $g$ stands for the gravitational acceleration. In the equations above and throughout this paper, the subscripts refer to the partial derivatives. These equations in the flow domain must be completed by kinematic and dynamic boundary conditions at the bottom and at the upper free surface. We have

$$
\begin{gathered}
w=0 \quad \text { at } z=0, \\
S_{t}+u S_{x}+v S_{y}-w=0 \quad \text { at } z=S(x, y, t), \\
p^{*}=p_{0}-T \frac{S_{x x}\left(1+S_{y}^{2}\right)+S_{y y}\left(1+S_{x}^{2}\right)-2 S_{x y} S_{x} S_{y}}{\left(1+S_{x}^{2}+S_{y}^{2}\right)^{3 / 2}} \\
\text { at } z=S(x, y, t) .
\end{gathered}
$$

Equation (2c) is the Laplace-Young boundary condition which rules the pressure difference between the two sides of the interface. We assume in Eq. (2c) that the pressure in the upper fluid (typically air) remains uniform and equal to $p_{0}$.

Shallow water equations are usually derived by performing an asymptotic analysis directly on the Euler equations (1) and boundary conditions (2). The velocity and the pressure fields are then handled perturbatively through the use of asymptotic expansions. Our approach is somewhat different since, instead of studying the entire problem via a perturbation theory, we are going to consider first the nonlinear evolution of a given initial ansatz for the velocity field. We assume indeed that $u$ and $v$ are independent of $z$, that is,

$$
\begin{aligned}
& u=u(x, y, t), \\
& v=v(x, y, t) .
\end{aligned}
$$

This ab initio given velocity profile, known as the columnarflow ansatz, can be justified from linear theoretical arguments or, even better, from direct visualization of the particle trajectories of a plane periodic wave in water of fairly depth [18]. It was introduced long ago by Green and Nagdhi [6-8], not in this form but in the rather different framework of the Cosserat surface theory (see references quoted in $[7,8]$ ). Besides, these profiles are also obtained through the classical shallow water analysis.

The columnar-flow ansatz enables us to derive equations involving only three fields, namely $u, v$, and $S$, and to eliminate the $z$ dependence. From Eqs. (1a) and (2a), we have

$$
w(x, y, z, t)=z q(x, y, t),
$$

where

$$
q(x, y, t)=-u_{x}(x, y, t)-v_{y}(x, y, t) .
$$

Equations (1b) and (1c) can then be integrated from $z=0$ to $z=S$ and, using Leibnitz's rule, we obtain

$$
\begin{aligned}
& \sigma \dot{u} S=-p_{x}-T \frac{a S_{x}}{b^{3 / 2}}, \\
& \sigma \dot{v} S=-p_{y}-T \frac{a S_{y}}{b^{3 / 2}},
\end{aligned}
$$

where the dot stands for the material derivative, i.e.,

$$
\dot{u}=u_{t}+u u_{x}+v u_{y},
$$

and the functions $p, a$, and $b$ are defined by

$$
\begin{gathered}
p(x, y, t)=\int_{0}^{S} p * d z-p_{0} S, \\
a(x, y, t)=S_{x x}\left(1+S_{y}^{2}\right)+S_{y y}\left(1+S_{x}^{2}\right)-2 S_{x y} S_{x} S_{y}, \\
b(x, y, t)=1+S_{x}^{2}+S_{y}^{2} .
\end{gathered}
$$

Next, we multiply Eq. (1d) by $z$ and integrate it from $z=0$ to $z=S$, which yields

$$
\sigma \frac{S^{3}}{3}\left(\dot{q}+q^{2}\right)+\sigma g \frac{S^{2}}{2}=p+T \frac{a S}{b^{3 / 2}} .
$$

The pressure $p$ may then be eliminated from Eqs. (7), (8), and (13), leading to

$$
\begin{aligned}
& \dot{u}=-S S_{x}\left(\dot{q}+q^{2}\right)-\frac{S^{2}}{3}\left(\dot{q}+q^{2}\right)_{x}-g S_{x}+\frac{T}{\sigma}\left(\frac{a}{b^{3 / 2}}\right)_{x}, \\
& \dot{v}=-S S_{y}\left(\dot{q}+q^{2}\right)-\frac{S^{2}}{3}\left(\dot{q}+q^{2}\right)_{y}-g S_{y}+\frac{T}{\sigma}\left(\frac{a}{b^{3 / 2}}\right)_{y},
\end{aligned}
$$

$$
\dot{S}=S q,
$$

and, substituting $q$ by its expression [Eq. (6)], we eventually obtain the equations involving the initial fields,

$$
\begin{aligned}
S\left(u_{t}+u u_{x}+v u_{y}\right)= & \frac{1}{3}\left[S ^ { 3 } \left(u_{x t}+u u_{x x}-u_{x}^{2}+v_{y t}+v v_{y y}-v_{y}^{2}\right.\right. \\
& \left.\left.+v u_{x y}+u v_{x y}-2 u_{x} v_{y}\right)\right]_{x}-g S S_{x} \\
& +\frac{T S}{\sigma}\left(\frac{a}{b^{3 / 2}}\right)_{x} \\
S\left(v_{t}+u v_{x}+v v_{y}\right)= & \frac{1}{3}\left[S ^ { 3 } \left(u_{x t}+u u_{x x}-u_{x}^{2}+v_{y t}+v v_{y y}-v_{y}^{2}\right.\right. \\
& \left.\left.+v u_{x y}+u v_{x y}-2 u_{x} v_{y}\right)\right]_{y}-g S S_{y} \\
& +\frac{T S}{\sigma}\left(\frac{a}{b^{3 / 2}}\right)_{y} \\
S_{t} & +(u S)_{x}+(v S)_{y}=0 .
\end{aligned}
$$

These equations constitute a Green-Nagdhi system, with surface tension, in $(2+1)$ dimensions. 
The linear dispersion relation $\omega(k, l)$ of the system $(15)$ is derived by expanding $u, v$, and $S$ into the following form $(\epsilon<1)$ :

$$
\begin{gathered}
u=\epsilon U+O\left(\epsilon^{2}\right), \\
v=\epsilon L+O\left(\epsilon^{2}\right), \\
S=h+\epsilon W+O\left(\epsilon^{2}\right),
\end{gathered}
$$

where $h$ is the unperturbed depth. At the order $\epsilon$, we obtain

$$
\begin{gathered}
U_{t}=\frac{1}{3} h^{2}\left(U_{x t x}+L_{y t x}\right)-g W_{x}+\frac{T}{\sigma}\left(W_{x x x}+W_{y y x}\right), \\
L_{t}=\frac{1}{3} h^{2}\left(U_{x t y}+L_{y t y}\right)-g W_{y}+\frac{T}{\sigma}\left(W_{x x y}+W_{y y y}\right), \\
W_{t}=-h\left(U_{x}+L_{y}\right) .
\end{gathered}
$$

To look for the possible solutions of the preceding linear system, we set $U=\alpha e^{i \phi}, L=\beta e^{i \phi}$, and $W=\gamma e^{i \phi}$, where

$$
\phi=k x+l y-\omega(k, l) t,
$$

$k$ and $l$ are the wave numbers in the $x$ and $y$ directions, respectively, and $\alpha, \beta$, and $\gamma$ are arbitrary constants. From Eq. (17), $\alpha, \beta$, and $\gamma$ obey a linear homogeneous system which has nontrivial solutions if

$$
\omega(k, l)^{2}=\left(l^{2}+k^{2}\right) \frac{g h+\frac{T h}{\sigma}\left(l^{2}+k^{2}\right)}{1+\frac{h^{2}}{3}\left(l^{2}+k^{2}\right)} .
$$

The Euler equations (1) and (2) have the dispersion relation

$$
\Omega^{2}=\left(k^{2}+l^{2}\right)^{1 / 2}\left(g+\frac{T\left(k^{2}+l^{2}\right)}{\sigma}\right) \tanh \left[\left(k^{2}+l^{2}\right)^{1 / 2} h\right] .
$$

We can compare the phase velocity $C$ and the group velocity $C_{g}$ associated with Eq. (20) with the analogous $c$ and $c_{g}$ associated with Eq. (19). This is done in Fig. 1 and Fig. 2 for $\Omega(k)$ and $\omega(k)(l=0)$. The pictures show that the linear model (15a), (15b), and (15c) approximates the linear Euler equations very well for $1<k h<2$. For $k h>2$, the divergence increases.

\section{ASYMPTOTIC MODEL FOR SHORT CAPILLARY-GRAVITY WAVES}

The system (15) can be seen as a first reduction of the Euler equations via the columnar-flow hypothesis. It incorporates finite dispersion not only in the long-wave limit but also in the short-wave one since the surface tension has been included in the model. Indeed, the dispersion relation is well behaved whether $\sqrt{k^{2}+l^{2}} \rightarrow 0$ (long waves) or $\sqrt{k^{2}+l^{2}} \rightarrow \infty$ (short waves). The long-wave case leads to the KP equation. To examine more precisely the short-scale behavior, we are

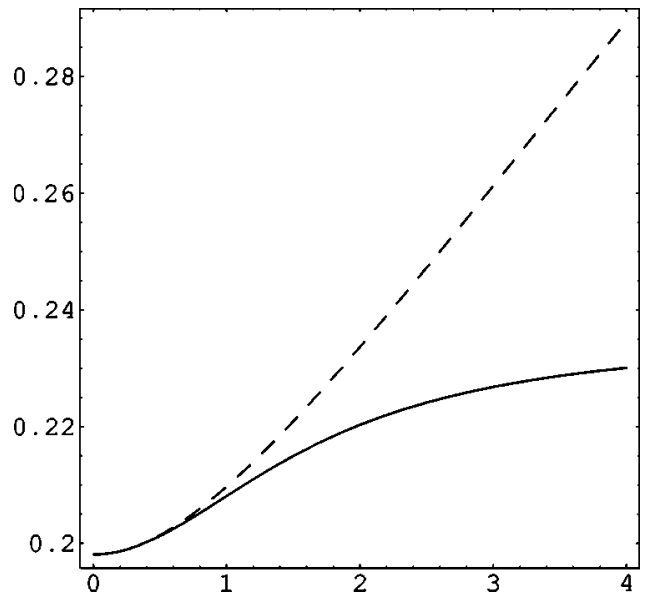

FIG. 1. Representation of the phase velocity $C(k)$ of Eq. (20) (broken line) and phase velocity $c(k)$ of Eq. (19) (continuous line) as a function of $k h$.

going to look for waves short in $x$ and normal in $y$, that is, to consider the $y$ dimension as being a lateral weak perturbation with respect to the $x$ one.

Two new variables are required to appropriately take into account short waves asymptotically in time: a spatial variable, $\zeta$, describing a local pattern and a temporal one, $\tau$, matching large times. To be compatible with the plane progressive wave solution of angular frequency (19), we introduce a small parameter $\epsilon$ and take

$$
k=\frac{k_{0}}{\epsilon} \quad \text { and } l=l_{0},
$$

where $\epsilon<1$ and $k_{0}$ and $l_{0}$ are of order 1 . The expansion of $\omega$ in terms of $\epsilon$ reads

$$
\omega=k_{0} \sqrt{\frac{3 T}{\sigma h}}\left[\frac{1}{\epsilon}+\epsilon\left(\frac{g \sigma}{2 T k_{0}^{2}}+\frac{l_{0}^{2}}{2 k_{0}^{2}}-\frac{3}{2 h^{2} k_{0}^{2}}\right)+O\left(\epsilon^{3}\right)\right] .
$$

It leads to

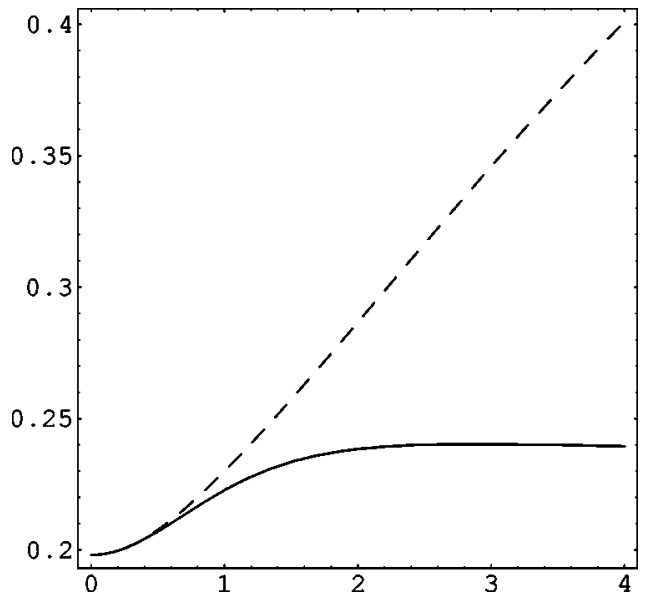

FIG. 2. Representation of the group velocity $C_{g}(k)$ of Eq. (20) (broken line) and group velocity $c_{g}(k)$ of Eq. (19) (continuous line) as a function of $k h$. 


$$
\zeta=\frac{1}{\epsilon}(x-V t), \quad y=y, \quad \tau=\epsilon t,
$$

where $V=\sqrt{3 T / \sigma h}$; the associated operators are given by

$$
\frac{\partial}{\partial x}=\frac{1}{\epsilon} \frac{\partial}{\partial \zeta}, \quad \frac{\partial}{\partial y}=\frac{\partial}{\partial y}, \quad \frac{\partial}{\partial t}=-\frac{V}{\epsilon} \frac{\partial}{\partial \zeta}+\epsilon \frac{\partial}{\partial \tau} .
$$

Now, using expressions (24) in Eqs. (15), together with the expansions

$$
\begin{gathered}
u=\epsilon^{2}\left(U_{0}+\epsilon^{2} U_{2}+\cdots\right), \\
v=\epsilon\left(L_{0}+\epsilon^{2} L_{2}+\cdots\right), \\
S=h+\epsilon^{2}\left(W_{0}+\epsilon^{2} W_{2}+\cdots\right),
\end{gathered}
$$

we can isolate nonlinear dynamics of short capillary-gravity waves from the system (15) by performing a perturbative calculation according to $\epsilon$.

Equations (15a) in order $1 / \epsilon$, Eq. (15b) in order $\epsilon^{0}$, and Eq. (15c) in order $\epsilon$ yield the following system:

$$
\begin{gathered}
V\left(U_{0, \zeta}+L_{0, y}\right)=\frac{3 T}{\sigma h^{2}} W_{0, \zeta}, \\
V L_{0}=\frac{1}{3} V h^{2}\left(U_{0, \zeta y}+L_{0, y y}\right)-\frac{T}{\sigma} W_{0, \zeta y}, \\
V W_{0, \zeta}=h\left(U_{0, \zeta}+L_{0, y}\right),
\end{gathered}
$$

whose solution is

$$
U_{0}=\frac{V}{h} W_{0}, \quad L_{0}=0 \quad \text { with } \quad V^{2}=\frac{3 T}{\sigma h} .
$$

Next, the orders $\epsilon$ of Eq. (15a) and $\epsilon^{3}$ of Eq. (15c) lead to an evolution equation for $U_{0}$ in $\zeta, y$, and $\tau$ coordinates. Rewritten with the initial variables, it reads

$$
u_{x t}=\frac{3 g}{2 V h}(1-3 \theta) u-\frac{1}{2} u_{x x} u-\frac{1}{4} u_{x}^{2}+\frac{3 h^{2}}{4 V} u_{x x} u_{x}^{2}-\frac{V}{2} u_{y y},
$$

where $u(x, y, t)$ is the fluid velocity at the surface and $\theta$ is a dimensionless parameter, the Bond number, given by

$$
\theta=\frac{T}{\sigma h^{2} g} .
$$

Equation (28) governs the nonlinear propagation of short waves in the long-wave model (15).

\section{ANALYSIS OF THE (1+1) TRAVELING-WAVE SOLUTIONS}

As a first investigation in the study of Eq. (28), one may begin by looking for its possible traveling-wave solutions. Owing to the difference between the space scales in each direction, we will only consider plane waves propagating in the short-scale one, i.e., we eliminate the $y$ dependence from Eq. (28) by removing the $u_{y y}$ term,

$$
u_{x t}=\frac{3 g}{2 V h}(1-3 \theta) u-\frac{1}{2} u_{x x} u-\frac{1}{4} u_{x}^{2}+\frac{3 h^{2}}{4 V} u_{x x} u_{x}^{2} .
$$

We then introduce the following dimensionless variables:

$$
u^{\prime}=\frac{\lambda}{3} \frac{u}{V}, \quad x^{\prime}=\frac{\lambda}{3} \frac{x}{h}, \quad t^{\prime}=\frac{V}{2 h} t,
$$

where $\lambda$ is a nonzero dimensionless parameter defined by

$$
\lambda=\frac{3(1-3 \theta)}{\theta} \text {. }
$$

They lead, if $\theta \neq 1 / 3$, to the more convenient form of Eq. (30) (dropping the primes),

$$
u_{x t}=u-u_{x x} u-\frac{1}{2} u_{x}^{2}+\frac{\lambda}{2} u_{x x} u_{x}^{2}
$$

If $\theta=1 / 3$, Eq. (30) is dispersionless and will not be studied here (see $[19,20]$ for a detailed analysis of the long-wave dynamics for this special value of $\theta$ ). A traveling wave may be described by a function

$$
u(r)=u(x-c t),
$$

where $c$ denotes the velocity of the wave. In the following, we only consider waves moving to the right, i.e., we assume $c>0$. According to Eq. (33), the wave profile $u(r)$ must then obey the ordinary differential equation

$$
\left(\frac{\lambda}{2} u_{r}^{2}-u+c\right) u_{r r}=-u+\frac{1}{2} u_{r}^{2} .
$$

For $\lambda \neq 0$, the study of Eq. (35) may be carried out by using the following change of variables:

$$
\begin{gathered}
X=\frac{\lambda}{2} u_{r}^{2}-u+c, \\
Y=u .
\end{gathered}
$$

If $\lambda \neq 1$, it leads to the first-order differential system

$$
\begin{gathered}
X X_{r}=(1-\lambda)\left(Y+\frac{c}{\lambda-1}\right) Y_{r}, \\
Y_{r}^{2}=\frac{2}{\lambda}(X+Y-c),
\end{gathered}
$$

which is equivalent to Eq. (35) provided one excludes from Eqs. (37) the solutions $Y_{r}=0$ with $Y \neq 0$. The phase portrait of the system (37) is then easily obtained since Eq. (37a) can be integrated, giving

$$
X^{2}+(\lambda-1)\left(Y+\frac{c}{\lambda-1}\right)^{2}=\lambda \kappa,
$$

where $\kappa$ is determined by the initial conditions. For $\lambda=1$, Eq. (37b) is still valid, but Eq. $\left(37 \mathrm{a}^{\prime}\right)$ now becomes $X^{2}+2 c Y=\kappa$.

In the following, we restrict the study of Eq. (35) to the solutions $u$ for which there exists $r_{0}$ such that $u_{r}\left(r_{0}\right)=0$ (the solution $u=0$ is left aside). We also define $u_{0}=u\left(r_{0}\right)$ and then have from Eq. $\left(37 \mathrm{a}^{\prime}\right) \kappa=u_{0}^{2}+c^{2} /(\lambda-1)$ if $\lambda \neq 1$ and $\kappa=u_{0}^{2}$ 

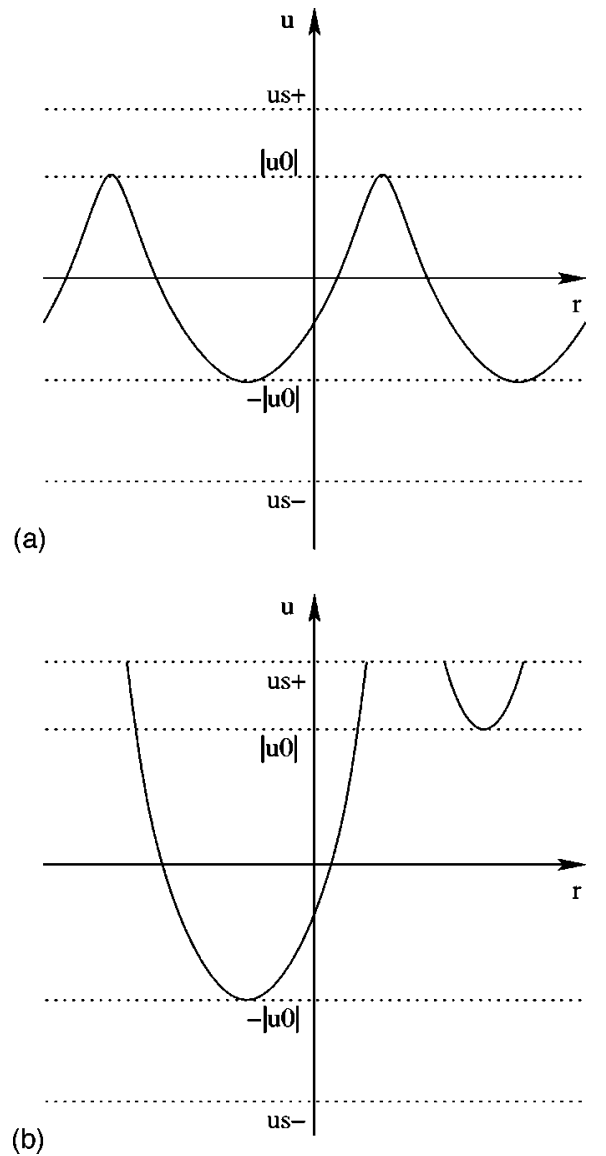

(b)

FIG. 3. Schematic representation of the traveling-wave solutions for $\lambda \geqslant 1 . u_{S}^{ \pm}$is defined for all values of $u_{0}$ and is such that $\left|u_{S}^{ \pm}\right|$ $\geqslant\left|u_{0}\right|$.

$+c^{2}$ if $\lambda=1$. We found two main cases when $\lambda>0$. If $\left|u_{0}\right|$ $<c$, the solutions are periodic and defined for all $r$ (Figs. 3 and 4). Otherwise, they show a singularity for a finite value of $r$. In the more specific case $0<\lambda<1$, the situation is actually a little more involved since the above singularity disappears when $\left|u_{0}\right|>c / \sqrt{1-\lambda}>c$ : the solution is then defined for all $r$ but is not periodic (i.e., not bounded). When $\lambda<0$, the solutions found are periodic again, similar to the previous ones, if $\left|u_{0}\right|<c / \sqrt{1-\lambda}$ (Fig. 5). They show a singularity if $u_{0}<-c / \sqrt{1-\lambda}$ or $c / \sqrt{1-\lambda}<u_{0}<c$ and are not bounded if $u_{0}>c$. The value of the function $u$ at the singularity point (see Figs. 3, 4, and 5 for further details) is given by

$$
u_{S}^{ \pm}=\frac{c}{1-\lambda} \pm \frac{1}{|\lambda-1|} \sqrt{\lambda\left[(\lambda-1) u_{0}^{2}+c^{2}\right]}
$$

if $\lambda \neq 1$ and $u_{S}=\left(u_{0}^{2}+c^{2}\right) /(2 c)$ if $\lambda=1$.

The preceding analysis shows that Eq. (33) has travelingwave solutions for all values of $\lambda(\lambda \neq 0)$. These waves share the same feature: they only exist if their amplitude $\left|u_{0}\right|$ is small enough. The threshold value $u_{C}$ depends on the sign of $\lambda: u_{C}(\lambda)=c$ if $\lambda>0$ and $u_{C}(\lambda)=c / \sqrt{1-\lambda}$ if $\lambda<0$. Below $u_{C}$, the wave is periodic and smooth, whereas as the amplitude reaches the threshold value, a singularity appears in the top
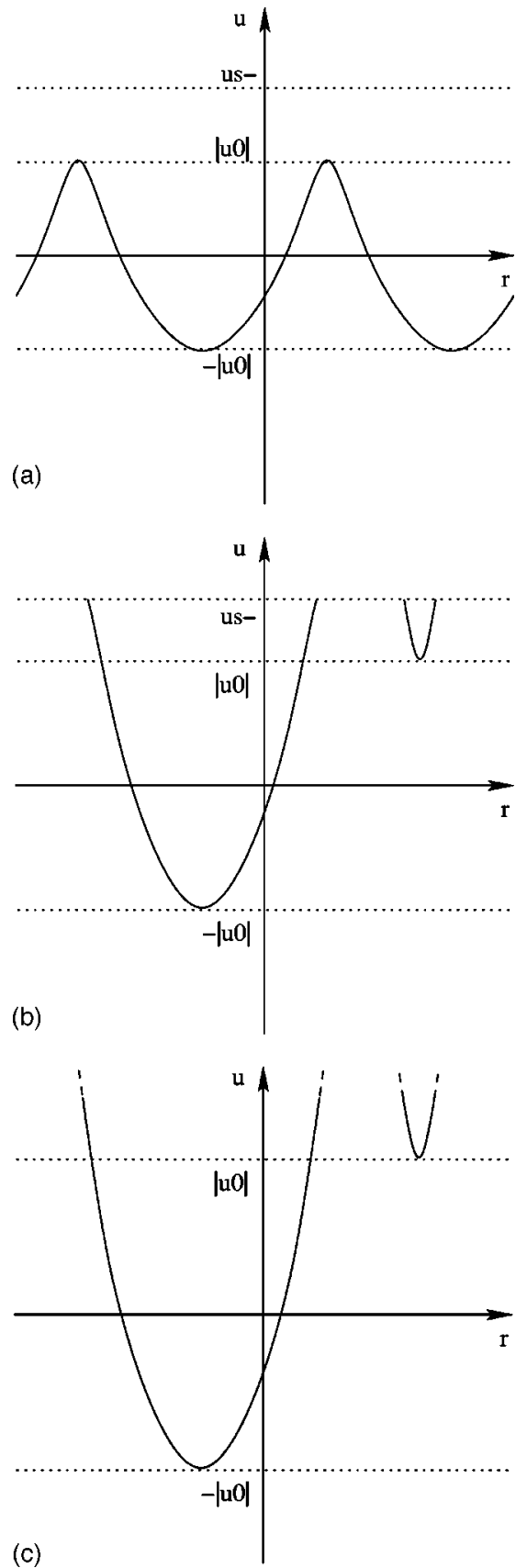

FIG. 4. Schematic representation of the traveling-wave solutions for $0<\lambda<1 . u_{S}^{ \pm}$is defined only when $\left|u_{0}\right| \leqslant c / \sqrt{1-\lambda}$; in that case, $u_{S}^{-} \geqslant\left|u_{0}\right|$.

part of the wave. Beyond the threshold, a solution smooth, bounded, and defined for all $r$ no longer exists. However, from the existing solutions of Eq. (35), one may build a periodic piecewise function which may be seen as the evolution of the periodic wave after it ceases to exist with a smooth shape, and whose only singularities are located at the crests, periodically distributed on the $r$ axis (Fig. 6). Actually, from a physical point of view such construction is relevant only when $u_{0}<0$. According to Eq. (38), the height of the crest is given by, if $\lambda \neq 1$, 

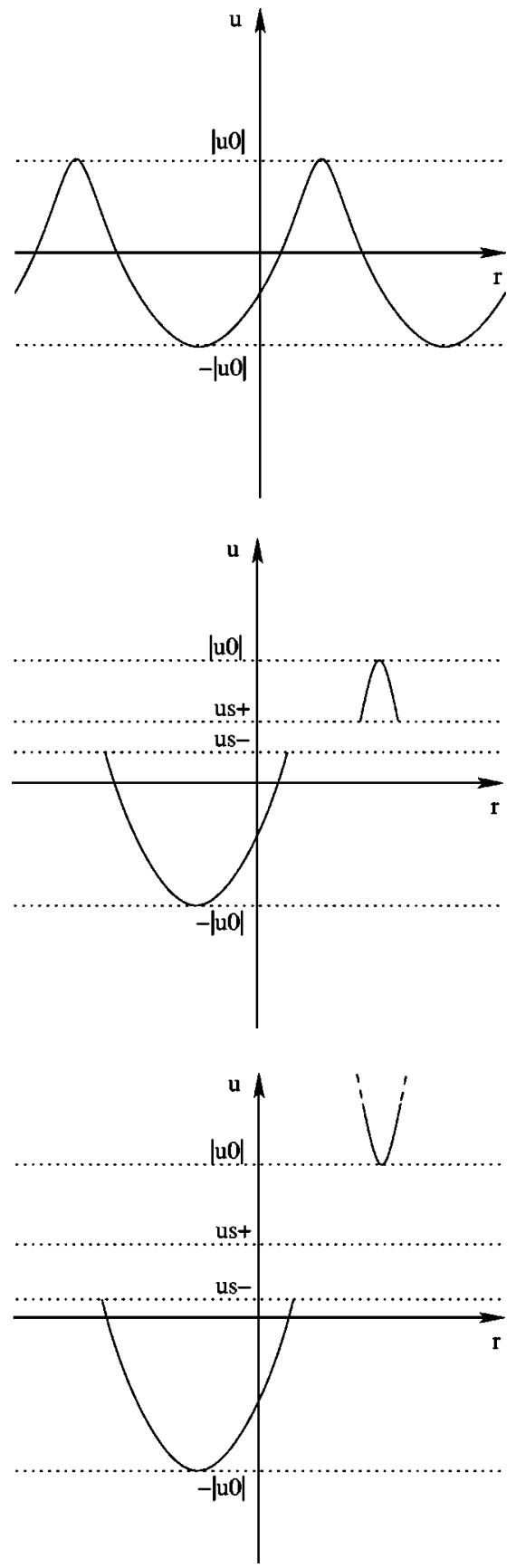

FIG. 5. Schematic representation of the traveling-wave solutions for $\lambda<0 . u_{S}^{ \pm}$is defined only when $\left|u_{0}\right| \geqslant c / \sqrt{1-\lambda}$; in that case, $u_{S}^{ \pm}$ $\leqslant\left|u_{0}\right|$.

$$
u_{S}\left(u_{0}, \lambda\right)=\frac{c}{1-\lambda}+\frac{1}{\lambda-1} \sqrt{\lambda\left[(\lambda-1) u_{0}^{2}+c^{2}\right]},
$$

provided it is defined, and $u_{S}\left(u_{0}, 1\right)=\left(u_{0}^{2}+c^{2}\right) /(2 c)$. It may be worth noting that if $\lambda<0$, the lower part of the resulting wave is more extensive than the upper one [i.e., $u_{S}\left(u_{0}, \lambda\right)$ $<-u_{0}$ ], whereas it is the opposite if $\lambda>0$ [i.e., $u_{S}\left(u_{0}, \lambda\right)>$ $-u_{0}$ if $\lambda>0$ ]. Provided it corresponds to the actual evolution of the smooth periodic wave, the piecewise function provides some insight into how the wave becomes singular. We can

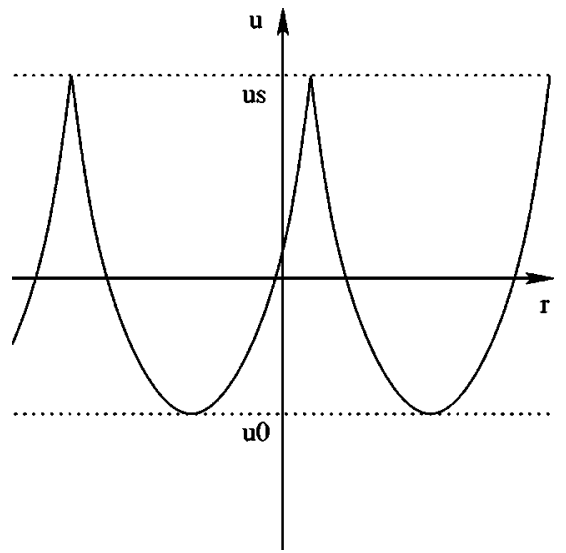

FIG. 6. Piecewise continuous function built with solutions of Eq. (35) for $\lambda>0$. It ranges from $u_{0}$ to $u_{S}$.

distinguish between two scenarios according to whether $\lambda$ is positive or not. In the former case, $u_{S}\left(u_{0}, \lambda\right)=-u_{0}$ when the threshold is reached $\left[-u_{0}=u_{C}(\lambda)\right]$ : the crests become more and more sharp as the amplitude of the wave increases until the top of the wave gives rise to a peak (Fig. 7). In the latter case, a breaking occurs in the middle of the wave since we have $u_{S}\left(u_{0}, \lambda\right)=-u_{0} / \sqrt{1-\lambda}$, and accordingly $u_{S}\left(u_{0}, \lambda\right)<-u_{0}$, when $-u_{0}=u_{C}(\lambda)$ (Fig. 8).

To complete this study, it is interesting to compute the angle of the wave shape at the crest for the critical case $u_{0}$ $=-u_{C}(\lambda)$. Provided it is defined, the slope at the break point reads

$$
\left|u_{1 S}^{\prime}\right|=\sqrt{\frac{2}{1-\lambda}\left(c-\frac{1}{\lambda} \sqrt{\lambda\left[(\lambda-1) u_{0}^{2}+c^{2}\right]}\right)}
$$

if $\lambda \neq 1$ and $\left|u_{1 S}^{\prime}\right|=\sqrt{\left(u_{0}^{2}-c^{2}\right) / 2}$ if $\lambda=1$. When $\lambda$ is negative, the substitution of $u_{0}$ by $-u_{C}$ yields $\left|u_{1 S}^{\prime}\right|=\sqrt{2 c /(1-\lambda)}$. In contrast, when $\lambda$ is positive, we obtain 0 , which is not surprising since the singularity in that case occurs at the wave crest. The slope of the wave shape at this point is then of little interest and we found it more convenient to evaluate the slope at the inflection point instead. We find (this expression is not valid when $\lambda<0$ and $-u_{0}>u_{C}$ )

$$
\left|u_{2 S}^{\prime}\right|=\sqrt{\frac{2}{1-\lambda}\left[c-\sqrt{(\lambda-1) u_{0}^{2}+c^{2}}\right]}
$$

if $\lambda \neq 1$ and $\left|u_{2 S}^{\prime}\right|=-u_{0} / \sqrt{c}$ if $\lambda=1$. It leads to, for $u_{0}=-u_{C}$,

$$
\left|u_{2 S}^{\prime}\right|=\sqrt{\frac{2 c}{1+\sqrt{\lambda}}} .
$$

These expressions show that the limit angle at the crest vanishes as $\lambda \rightarrow \infty$ (i.e., $T \rightarrow 0$ ) while, when $\lambda=-9$ (i.e., $T \rightarrow \infty$ ), it remains finite.

\section{MODULATIONAL INSTABILITY}

In this section, we study the resonant interaction occurring in a wave train (Stokes' wave train) with a narrow band of frequencies and wavelengths. Let us consider $u(x, t)$ as a 


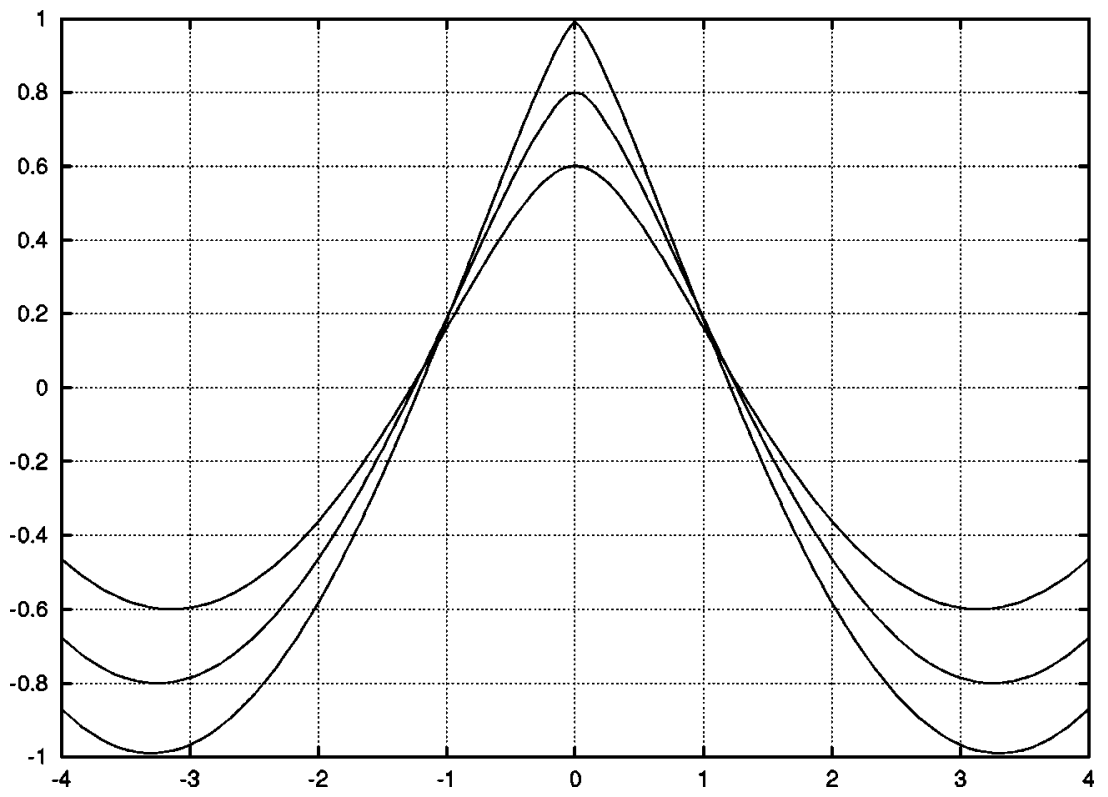

FIG. 7. Numerical integration of Eq. (35) for $\lambda=2$ and $c=1$. The crest of the wave becomes sharper as the wave amplitude $-u_{0}$ approaches the critical value $u_{C}(\lambda)=c$. The values of $-u_{0}$ for the three curves are $0.8,0.9$, and 0.99 .

plane wave. The nonlinear terms in Eq. (30) give rise to harmonics of the fundamental. Assume that a disturbance is present consisting of modes with sideband frequences and wave numbers close to the fundamental. We can have interaction between harmonics and these sideband modes. This interaction is likely to produce a resonant phenomenon manifesting itself by the modulation of the plane-wave solution. The exponencial growth in time of the modulation, originating from synchronous resonance between harmonics and sideband modes, leads to the Benjamin-Feir instability [21]. A formal solution can be given via an asymptotic expansion leading to the nonlinear Schrödinger equation (NLS) [22]. The particular interest of NLS in the existence of a general and simple criterion enables us to detect the stability or instability of the monochromatic wave train. Let us seek a solution of Eq. (30) under the form of a Fourier expansion in harmonics of the fundamental exp $i\left(k x-\omega_{s} t\right)$ and where the Fourier components are developed in a Taylor series in pow- ers of a small parameter $\delta$ measuring the amplitude of the fundamental,

$$
u=\sum_{p=1}^{\infty} \sum_{l=-p}^{l=p} \delta^{p} u_{l}^{p}(\xi, \tau) \exp \left[i l\left(k x-\omega_{s} t\right)\right]
$$

Eq. (43), $u_{-l}^{p}=u_{l}^{* p}$ (the asterisk denotes complex conjugation) and $\xi$ and $\tau$ are slow variables introduced through the stretching $\xi=\delta(x-C t)$ and $\tau=\delta^{2} t$ and where $C$ will be determined as a solvability condition. The expansions (43) include fast local oscillations through the dependence on the harmonics and slow variation (modulation) in amplitude taken into account by the $\xi, \tau$ dependence of $u_{l}^{p}$. Introducing now this expansion and the slow variables in Eq. (30), we may proceed to collect and solve different order $\delta$ and $l$. We have with

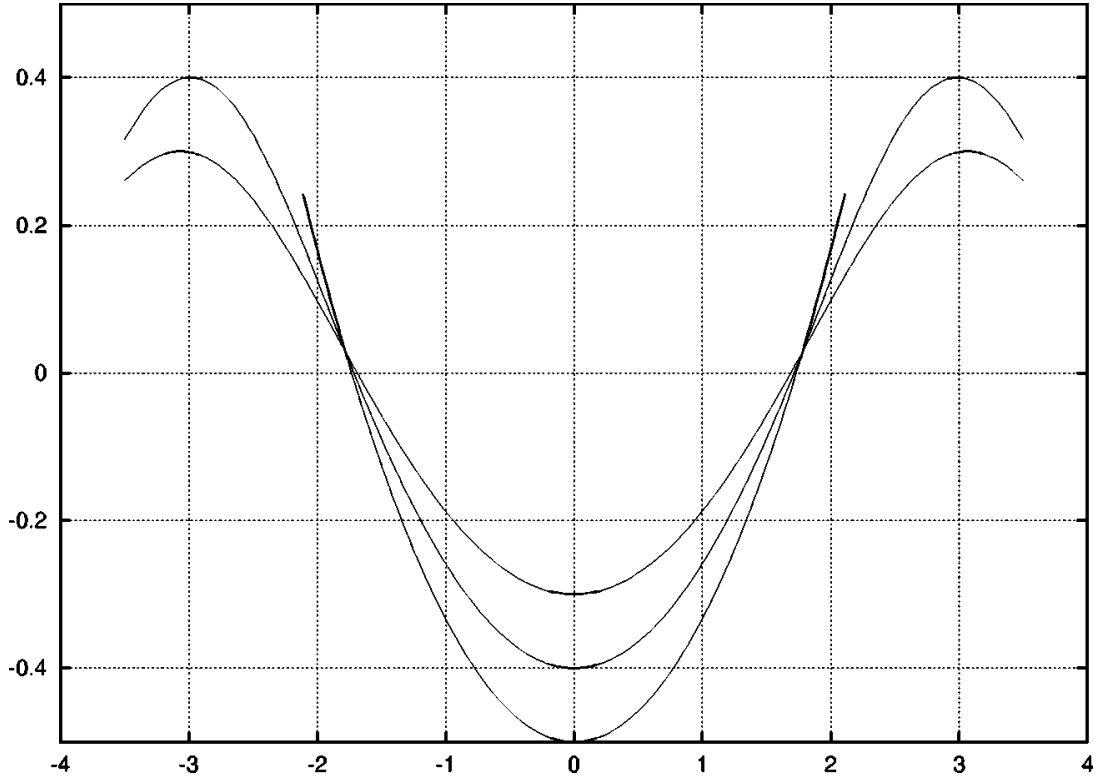

FIG. 8. Numerical integration of Eq. (35) for $\lambda=-3$ and $c=1$. As the wave amplitude $-u_{0}$ reaches the critical value $u_{C}(\lambda)=c / \sqrt{1-\lambda}$, the wave breaks at $u_{S}\left(-u_{C}(\lambda), \lambda\right)=c /(1-\lambda)$. The values of $-u_{0}$ for the three curves are $0.3,0.4$, and 0.50001 . 


$$
u_{1}^{1}=\psi(\xi, \tau), \quad A=\frac{3 g(1-3 \theta)}{2 V h}, \quad B=\frac{3 h^{2}}{4 V},
$$

the following conditions of solvability:

$$
\begin{gathered}
u_{0}^{1}=0, \quad \omega_{s}=k V+\frac{A}{k}, \quad C=2 V-\frac{\omega_{s}}{k}, \\
u_{0}^{2}=-\frac{k^{2}}{2 A}|\psi|^{2}, \quad u_{2}^{2}=\frac{k^{2}}{4 A} \psi^{2}, \quad u_{1}^{2}=\frac{i}{k} \psi_{\xi} .
\end{gathered}
$$

At order $\delta=3, l=1$ we obtain as a solvability condition the NLS for $\psi(\xi, \tau)$,

$$
-i \psi_{\tau}-\frac{A}{k^{3}} \psi_{\xi \xi}+\left(\frac{1}{8 A}-B\right) k^{3} \psi|\psi|^{2}=0 .
$$

The nature of solutions of NLS depends drastically on the sign of the product between the coefficient of $\psi_{\xi \xi}$ and that of $\psi|\psi|^{2}$. In this case, this product is positive for

$$
\theta<\frac{3}{10}
$$

and according to a well known stability criterion (see, for example, [1]), Stokes' wave train is unstable, that is, any slight deformation of the plane wave experiences an exponential growth. In the case of water at room temperature $(T$ $=0.074 \mathrm{~N} \mathrm{~m}^{-1}, \sigma=10^{3} \mathrm{~kg} \mathrm{~m}^{-3}$ ), we obtain that a short-wave train is unstable for a depth $h>0.49 \mathrm{~cm}$.

Last but not least, the value $\theta=3 / 10$ corresponds to $\lambda$ $=1$ in Eq. (33). Precisely,

$$
\theta<0.3 \Rightarrow \lambda>1
$$

\section{FINAL REMARKS}

The purpose of this paper was to investigate the behavior of short waves in a long-wave model. The presence of these waves may have different origins. First, in modeling real physical systems, we are often led to use these models beyond the precise range of validity under which they were derived. In that situation, we will very probably be in a shortwave region. Second, whenever we realize numerical discretizations of them, short waves are introduced as secondary effects coming from truncations and finite-difference methods, when the wavelength is of the order of the grid spacing. Third, initial wave-packet solutions, in terms of Fourier integrals, contain in general short-wave components. Instabilities of these waves can cause instability in the entire system. In this paper, we have investigated the short-wave dynamics in a Green-Naghdi model with surface tension. We have found that the traveling plane-wave solutions exist for all the values of the surface tension parameter (except $\theta=1 / 3$ ) and have determined an amplitude threshold beyond which these waves become singular. The limit angle value of the resulting wave is supplied. We have also studied the Benjamin-Feir instability and have specified the regions in which a wave train is modulationally stable or unstable.

Some points remain to be developed. Equation (30) was already studied in Ref. [23] in relation to its integrability and the solutions going to zero for $x \rightarrow \infty$. It has been shown that in that case the system is completely integrable and connected with the sine-Gordon or the sinh-Gordon equations depending on the value of $\lambda$. It would be interesting to know if this property is still valid for Eq. (28).

Finally, let us note that the study of wave dynamics at short scales in real fluids constitutes an open problem signifiant for theoretical or practical reasons. Dissipative phenomena take place at small scales. They ultimately appear from the turbulent motion of the fluid. The turbulent fluctuations of the fluid tend to drain energy and momentum from large scales of motion to short scales of motion where viscosity can act directly. Equation (28) was derived under the hypothesis that the system is dissipation-free. However, viscosity cannot always be neglected in real fluids, and this will certainly affect the asymptotic dynamics of short waves in longwave models and their stability. The solution of this problem must enlighten our knowledge of turbulence phenomena.

\section{ACKNOWLEDGMENTS}

C.H.B. and M.A.M. wish to thank the IFT-UNESP for their hospitality and FAPESP-Brasil for financial support.
[1] G. B. Whitham, Linear and Nonlinear Waves (Wiley Interscience, New York, 1974).

[2] R. K. Dodd, J. C. Eilbeck, J. D. Gibbon, and H. C. Morris, Solitons and Nonlinear Wave Equations (Academic Press, London, 1982).

[3] E. Infeld and G. Rowlands, Nonlinear Waves, Solitons and Chaos (Cambridge University Press, Cambridge, 1990).

[4] D. H. Peregrine, J. Fluid Mech. 25 (Pt. 2), 321 (1966).

[5] T. B. Benjamin, J. L. Bona, and J. J. Mahony, Philos. Trans. R. Soc. London, Ser. A 272, 47(1972).

[6] A. E. Green, N. Laws, and P. M. Nagdhi, Proc. R. Soc. London, Ser. A 338, 43 (1974).

[7] A. E. Green, and P. M. Nagdhi, J. Fluid Mech. 78, 237 (1976).
[8] A. E. Green, and P. M. Nagdhi, Proc. R. Soc. London, Ser. A 347, 447 (1976).

[9] M. A. Manna and V. Merle, Proc. R. Soc. London, Ser. A 454, 1445 (1998).

[10] M. A. Manna, J. Phys. A 34, 4475 (2001).

[11] S. M. Gama, R. A Kraenkel, and M. A. Manna, Inverse Probl. 17, 863 (2001).

[12] M. W. Dingemans, Report No. R 729-H, Delft Hydr. Lab, Delft, The Netherlands (1973).

[13] J. P. Broer, Appl. Sci. Res. 32, 619 (1976).

[14] P. Van Der Houwen, P. J. Mooiman, and F. W. Wuls, Int. J. Numer. Methods Fluids 13 (10), 1235 (1991).

[15] N. D. Katopodes, B. F. Sanders, and J. P. Boyd, J. Waterw., 
Port, Coastal, Ocean Eng. 124, 5 (1998); 124, 238 (1998).

[16] M. A. Manna and V. Merle, Phys. Rev. E 57, 6206 (1998).

[17] R. A. Kraenkel, M. A. Manna, and V. Merle, Phys. Rev. E 60, 2418 (1999).

[18] M. Van Dyke, An Album of Fluid Motion (The Parabolic Press, Stanford, CA, 1997).

[19] T. B. Benjamin, Q. Appl. Math. 39, 231 (1982).
[20] J. K. Hunter and J.-M. Vanden-Broeck, J. Fluid Mech. 134, 205 (1983).

[21] T. B. Benjamin and J. E. Feir, J. Fluid Mech. 27, 417 (1967).

[22] J. T. Stuart and R. C. Di Prima, Proc. R. Soc. London, Ser. A 362, 27 (1978).

[23] M.A. Manna and A. Neveu, e-print physics/0303085 (unpublished). 